\title{
Association between breastfeeding duration and cognitive development, autistic traits and ADHD symptoms: a multicenter study in Spain
}

\author{
Olivier Boucher ${ }^{1}$, Jordi Julvez 2,3,4,5 , Mònica Guxens ${ }^{2,4,6,7}$, Enrique Arranz ${ }^{8,9}$, Jesús Ibarluzea ${ }^{4,8,10}$, Manuel Sánchez de Miguel ${ }^{9}$, \\ Ana Fernández-Somoano 4,11 , Adonina Tardon ${ }^{4,11}$, Marisa Rebagliato ${ }^{4,12,13}$, Raquel Garcia-Esteban ${ }^{2,4,6}$, Giselle O'Connor ${ }^{2,6}$, \\ Ferran Ballester ${ }^{4,13}$ and Jordi Sunyer $2,3,4,6$
}

BACKGROUND: Several studies have related longer breastfeeding duration to better intellectual performance in children. By contrast, few studies have investigated the potential protective effects of breastfeeding against behavioral problems such as attention deficit hyperactivity disorder (ADHD) symptoms, and even fewer on autism spectrum disorders (ASD) traits.

METHODS: We examined the association between breastfeeding duration and cognitive development, attention, ADHD symptoms, and autistic traits using data from the INMA Project, a Spanish multicenter birth-cohort study, and taking into account the intensity of breastfeeding. Duration of any, predominant, and exclusive breastfeeding was documented during infancy through maternal questionnaires. Children ( $N$ $=1,346$; mean age $=4.9$ y) were assessed using the McCarthy Scales of Children's Abilities, Conners' Kiddie Continuous Performance Test, criteria of the DSM-ADHD symptoms form list, and the Childhood Autism Spectrum Test.

RESULTS: After adjustment for several confounders, longer duration of breastfeeding was independently associated with better cognitive development and with fewer autistic traits.

CONCLUSION: This study provides further evidence of a positive association of breastfeeding with cognitive function apart from socio-environmental factors, and also suggests a protective role against autistic traits. Results are in agreement with recommendations for prolonged breastfeeding duration to promote child development.

B reastfeeding has been associated with better intellectual function in children in several epidemiological studies (1). Confounding by parental and socio-environmental factors such as maternal IQ, smoking during pregnancy, parental education, and quality of home environment constitutes a major issue in these studies, since this association may be reduced to a nonsignificant trend after statistical control for such variables (2). Nevertheless, the majority of recent investigations have supported the existence of an association between duration of breastfeeding and child cognition beyond parental and socio-environmental factors (3-6). Inconsistent assessment of breastfeeding duration and intensity, and variability in the tasks used to assess child intelligence, may have accounted for negative findings in some studies $(7,8)$.

In comparison to the large amount of literature relating breastfeeding to intellectual function in children, very few studies have assessed its potential effects on symptoms of attention deficit hyperactivity disorder (ADHD) and pervasive developmental disorders. Some epidemiological investigations have examined the associations between breastfeeding and behavioral inattention and/or hyperactivity, with some suggesting protective effects $(9,10)$, but others not $(11,12)$. The use of a general questionnaire of children's strengths and difficulties rather than a specific ADHD questionnaire, and the lack of direct assessment of children's attention function, may account for the absence of significant association in the latter studies. Other factors, such as the different categorizations of breastfeeding duration and intensity, further complicate comparisons between studies. A few retrospective studies have reported that children with autism spectrum disorders (ASD) are typically breastfed for shorter periods than normally-developing children (13-15). However, a recent study using data from a large representative survey of US children found no relationship between breastfeeding history and ASD diagnosis (16). To our knowledge, no prospective study has yet examined the relationship of breastfeeding with autistic traits and ASD.

In Spanish children involved in the sub-cohort from Sabadell of the Infancia y Medio Ambiente (INMA) -Environment and Childhood-Project, we reported that prolonged duration of predominant breastfeeding was independently associated with

\footnotetext{
'Département de psychologie, Université de Montréal, Montréal, Québec, Canada; ${ }^{2}$ Institut de Salut Global de Barcelona (ISGlobal), Barcelona, Spain; ${ }^{3}$ Hospital del Mar Research Institute (IMIM), Barcelona, Spain; ${ }^{4}$ Spanish Consortium for Research on Epidemiology and Public Health (CIBERESP), Madrid, Spain; ${ }^{5}$ Carlos III Health Institute, Madrid, Spain; ${ }^{6}$ Pompeu Fabra University, Barcelona, Catalonia, Spain; ${ }^{7}$ Department of Child and Adolescent Psychiatry/Psychology, Erasmus University Medical Centre-Sophia Children's Hospital, Rotterdam, The Netherlands; ${ }^{8}$ BIODONOSTIA, Instituto de Investigación Biosanitaria, San Sebastián, Spain; ${ }^{\circ}$ Facultad de Psicología, UPV-EHU. San Sebastián, Basque Country, Spain; ${ }^{10}$ Subdirección Salud Publica Gipuzkoa, Basque Country, Spain; ' ${ }^{1}$ Department of Preventive Medicine and Public Health, University of Oviedo, Oviedo, Spain; ${ }^{12}$ University Jaume I, Castellón, Spain; ${ }^{13} \mathrm{FISABIO}-U J$ I-University of Valencia Joint Research Unit, Valencia, Spain. Correspondence: Jordi Julvez (jordi.julvez@isglobal.org) Received 17 March 2016; accepted 19 September 2016; advance online publication 4 January 2017. doi:10.1038/pr.2016.238
} 
cognitive function at $4 \mathrm{y}$ of age, after statistical control for a wide range of family and social variables, including parental mental health and maternal cognitive function (5). The present study assessed whether any, predominant, and exclusive breastfeeding duration have an impact on cognitive development, attention function, ADHD symptoms, and autistic traits, after taking into account parental and socio-environmental factors, and examines whether the impacts on cognitive development and on behavioral problems are independent from each other.

\section{METHODS}

\section{Participants and Procedure}

Participants were recruited as part of the INMA Project, a multicenter birth cohort study aimed at investigating the effect of environmental exposures and diet during pregnancy on fetal and child development in different areas of Spain (17). Cohorts included in this analysis were established in 2003-2008 in the geographical areas of Asturias, Gipuzkoa, Sabadell, and Valencia. A total of 2,644 eligible women ( $\geq 16 \mathrm{y}$, intention to deliver at the reference hospital, ability to communicate in Spanish or the other official language in the region, singleton pregnancy, no assisted conception) were recruited during prenatal visits in the first trimester of pregnancy. Women were followed through pregnancy and children from birth through the fourth year of life. Of the 2,506 children followed through birth, 2,080 (83\%) were evaluated at $\geq 4 \mathrm{y}$. Participants lost to follow-up were characterized by lower scores on most socio-environmental variables and had significantly shorter duration of exclusive breastfeeding in comparison to children still participating at $\geq 4$ y (Supplementary Table S8 online). Final analyses included all children $(N=1346)$ born at term ( $\geq 37 \mathrm{wk}$ ) with complete data for any breastfeeding variable, for whom assessment of cognitive development, attention, ADHD symptoms, or autistic traits was available, and for whom for all covariates were available. Participants who were excluded due to missing data on any confounding variable were younger at testing and their mothers had higher social class compared to the included participants, but they were comparable with breastfeeding duration and on the vast majority of confounding variables (Supplementary Table S9 online). Participating mothers signed an informed consent form in each phase, and the ethics committees of the centers involved approved the research protocol.

\section{Breastfeeding Assessment}

Detailed information about the child's diet was obtained using interviewer-administered questionnaires with the mothers when children were 6 mo old (Asturias and Sabadell sub-cohorts), 14 mo, and 4 y (all sub-cohorts) (Supplementary Figure S1 online). "Any breastfeeding" was defined as the child receiving any type of breast milk feeding, no matter if combined or not with other diets; "predominant breastfeeding", as the child breastfed only, but allowing supplementations of non-milk liquids, fruit juice, oral rehydration salts solution, drops, and syrup forms of vitamins, minerals, and medicines; and "exclusive" breastfeeding, as the child receiving breast milk only, not allowing other food or drink, not even water, but allowing the infant to receive oral rehydration salts solution, drops and syrups. Information on exclusive breastfeeding was not collected in the Valencia cohort $(n$ $=484,36.0 \%)$.

\section{Child Assessment}

Cognitive development was assessed using the Spanish version of the McCarthy Scales of Children's Abilities (MSCA) (18). The MSCA is composed of subtests assessing verbal, perceptive-performance, memory, quantitative, and motor function, and generates a General Cognitive Index (GCI). For each cohort, raw scores from the GCI were centered to a mean of 100 , with a standard deviation of 15 to compute index scores. All testing was performed by trained psychologists. Examiners identified children whose tests were of good quality $(n=1,265)$, of poor quality (e.g., due to fatigue, illness, etc.; $n=66)$ and those for whom testing was invalided due to lack of collaboration $(n=7)$. Children with invalid testing were excluded from all MSCA analyses.

Attention function was assessed using Conners' Kiddie Continuous Performance Test (K-CPT), a 7-min computerized test in which children were instructed to press the space-bar when they saw any image on the computer screen, except a ball (19). Omissions (child failing to respond when he/she should), false alarms (child responds when he/she should not) and hit reaction time (mean response time for all correct hits) were computed and examined separately.

ADHD symptoms were estimated using the ADHD criteria of Diagnostic and Statistical Manual of Mental Disorders, Fourth Edition (ADHD-DSM-IV) form list completed by the child's classroom teacher (20). ADHD-DSM-IV is an internationally recognized form list comprising 18 symptoms and is designed to evaluate attention deficit ( 9 symptoms) and hyperactivity and impulsivity ( 9 symptoms) in children. Each symptom is rated using a 4-point scale, i.e., 0 = "not at all", 1 = "just a little", 2 = "pretty much", and 3 = "very much". Sum of scores was calculated to provide continuous measures of inattention, hyperactivity/impulsivity, and combined ADHD symptoms. $\mathrm{ADHD}$ in the clinical range was estimated by recording ratings of options 0 and 1 as "symptom absent", and ratings of 2 and 3 as "symptom present", and using the DSM-IV criteria for ADHD ( $\geq 6$ symptoms within a single category). Cronbach's Alpha Coefficient analyses in our sample were $>0.89$ for all ADHD-DSM-IVscales, suggesting excellent internal consistency.

Autistic traits were assessed using the Spanish version of the Childhood Autism Spectrum Test (CAST; formerly Childhood Asperger Syndrome Test) (21). The CAST is a 37-item questionnaire that the evaluator administers to the child's parents. Each question can be scored with 0 (autism-negative response) or 1 point (autismpositive response), except for six questions that are not scored. The maximum possible score is 31 points. The total number of points was computed to provide a continuous measure of autistic traits. Higher scores indicate more autistic traits. Additionally, a score of 15 has been used as a cut-off to identify children at risk for ASD. Cronbach's Alpha Coefficient analysis in our sample was of 0.64 for CAST total score.

\section{Other Variables}

A set of potentially confounding variables was selected a priori based on prior studies of the effects of breastfeeding on child development. Information on parental education, social class, and other sociodemographic characteristics and habits (including maternal age and country of birth, parity, and maternal smoking) were obtained through questionnaires during the first and third trimesters of pregnancy, and when the child was 14 mo and 4 y of age (5). Child care attendance (yes/no) was assessed at each postnatal visit. Maternal verbal IQ was estimated at the 4-y assessment using the Similarities subtest of the Weschler Adult Intelligence Scales-Third Edition (WAIS-III), a subtest showing strong correlations with the full verbal scale (22). Raw scores were converted to an IQ-scale with a mean of 100 and a standard deviation of 15 , using data from the entire sample. Maternal psychopathology was assessed at the 4-y assessment using the Symptom Checklist - Revised (SCL-90-R), a self-reported questionnaire comprising 90 items which provides a Global Severity Index designed to measure overall psychological distress (23). T-scores $>65$ on the Global Severity Index were considered at risk for psychopathology. For the subsample of participants from the Gipuzkoa cohort, quality of family context was also assessed at 2 y using the Etxadi-Gangoiti Scale (EGS), a 127-item interview carried out in the family's home by psychologists. The EGS provides three subscales (Stimulation of cognitive and linguistic development, Stimulation of social and emotional development, and Organisation of the social context and physical environment) (24).

\section{Statistical Analyses}

The normality of each variable's distribution was inspected visually and checked for skewness and kurtosis (normality range: -2.0 to 2.0). Log transformations were conducted on each ADHDDSM-IV symptom variable and on K-CPT omission errors, since these variables followed log-normal distributions. Associations 
between breastfeeding duration and MSCA - GCI, K-CPT omission errors, commission errors and hit reaction time, ADHD-DSM-IV Inattention, Hyperactivity, and Total symptoms, and CAST total score were examined using linear regression analyses. Associations with ADHD scores within the clinical range and CAST scores in the borderline range (yes vs. no) were also examined using logistic regression analysis. In order to illustrate the extent to which socioenvironmental variables accounted for the associations between breastfeeding duration and outcomes, child and assessment covariates were included as a first step in the regression model (minimally-adjusted models), and social and parental covariates were entered as a second step (fully-adjusted models). Because birthweight and maternal age might not be linearly associated with child outcomes, several categories of these covariates were created, and those that explained the largest percentages of child outcomes were used in subsequent models. Standardized regression coefficients are reported to facilitate comparisons across developmental domains, and raw regression coefficients are also reported to facilitate interpretation of the results.

Minimally-adjusted models included the following child variables: age (continuous), sex, cohort (Asturias/Gipuzkoa/Sabadell/ Valencia), birthweight $(<-1.5 \mathrm{SD} /-1.5-0 \mathrm{SD} / 0-1.5 \mathrm{SD} />1.5$ $\mathrm{SD}$ to the sample mean), and quality of assessment (good vs. poor; MSCA analyses only). Fully-adjusted models additionally adjusted for parity ( 0 vs. $\geq 1)$, maternal variables: age $(<25 / 25-$ $29 / 30-34 / 35+$ years), education (primary or less/secondary school/ university), social class (managers, technicians, and associate professionals/skill manual and other nonmanual workers/semiskilled and unskilled manual workers), country of birth (Spain: yes/no), smoking during pregnancy (yes/no), verbal IQ proxy (continuous), and psychological distress (at risk for psychopathology: yes/no); paternal variables: education and social class (same categories as for maternal variables); and child care (attending to kindergarten at $18 \mathrm{mo}$ : yes/no). In order to examine whether different associations were observed for "any", "predominant" and "exclusive" breastfeeding due to sample differences, analyses were rerun including children with available data for all types of breastfeeding only. To exclude the possibility that any observed effect was attributable to differences between breastfed and nonbreastfed children, all analyses were also rerun without nonbreastfed children. Significant associations between duration of any breastfeeding and child outcomes were explored further by replacing the continuous breastfeeding duration variable by the following categories: 0 (not breastfed) -2 mo (used as reference); $2-6 \mathrm{mo} ; 6-12 \mathrm{mo}>12 \mathrm{mo}$. The effect of child gender on the relationship between duration of breastfeeding and each outcome was examined using linear regression analyses stratified by sex, and by adding the interaction term to the main regression models. Because quality of family context was available for $<20 \%$ of the sample, it was not included in the main regression models; nevertheless, because of previous evidence suggesting that quality of home environment constitutes a major confounding factor in the relationship between breastfeeding and child development (2), supplemental analyses were conducted in order to examine the impact of controlling for EGS scores on the study results, within the subsample from the Gipuzkoa sub-cohort for which this variable was available. In order to maximize sample size, this supplemental set of analyses only controlled for child characteristics in addition to quality of family context, which allowed for the inclusion of additional children who had missing data on socio-environmental variables. In order to examine whether the relationship of breastfeeding duration to a given child outcome was independent from its association with other child outcomes that were found to be associated with breastfeeding, all significant final models were rerun including the other outcomes as covariates. Finally, to evaluate the role of participant loss due to attrition and missing data, main linear regression models for each child outcome with duration of any breastfeeding as a predictor were reconducted using inverse probability weighting (25).

Associations were considered statistically significant at $P<0.05$. All analyses were conducted with IBM SPSS Statistics Version 22.0 (IBM, Armonk, NY).

\section{RESULTS}

\section{Descriptive Characteristics}

Descriptive characteristics of the total sample are presented in Table 1. A large majority of mothers breastfed their children, and those who breastfed did so for $7 \mathrm{mo}$ on average. About $90 \%$ of those mothers used a predominant breastfeeding diet for their child. Duration of each type of breastfeeding (any, predominant, and exclusive) was strongly correlated $(r: 0.60-$ 0.87 ). Several factors were simultaneously correlated with breastfeeding duration and child outcomes (Supplementary Table S1 online). Most importantly, parental education and social class, and maternal verbal IQ correlated with longer duration of breastfeeding and with better outcomes, whereas maternal psychopathology and smoking during pregnancy correlated with shorter breastfeeding duration and with poorer outcomes.

\section{Associations Between Breastfeeding Duration and Child Outcomes}

After adjustment for all covariates, longer duration of any, predominant, and exclusive breastfeeding were all associated with a small but significant improvement in cognitive development (MSCA), and longer duration of any and predominant breastfeeding were significantly associated with decreased autistic traits (CAST; Table 2). Additionally, longer duration of any and exclusive breastfeeding were associated with slower reaction time on the K-CPT. None of the associations between breastfeeding duration and ADHD symptoms remained significant in fully-adjusted models. Logistic regression analyses with the same set of covariates did not reveal any significant association between duration of any (odds ratio (OR): 0.97; 95\% confidence interval (CI): 0.92, 1.02)), predominant (OR: 0.92; 95\% CI: 0.82, 1.04), or exclusive (OR: 0.88; 95\% CI: 0.74, 1.04) breastfeeding and ADHD score within the clinical range, nor between duration of any (OR: $1.00 ; 95 \%$ CI: $0.95,1.05$ ), predominant (OR: 0.98 ; $95 \%$ CI: $0.87,1.10)$, or exclusive (OR: 1.02; 95\% CI: $0.86,1.22$ ) breastfeeding and CAST scores in the borderline range.

When restricting analyses to children with available data for duration of any, predominant, and exclusive breastfeeding to facilitate comparisons across intensities of breastfeeding, the strengths of the associations with child outcomes were highly similar, with the exception of autistic traits which were more strongly and only significantly associated with duration of any breastfeeding (Supplementary Table S2 online).

After exclusion of nonbreastfed children from the analyses, associations with cognitive development and with autistic traits remained significant and were even slightly strengthened when compared with analyses involving the whole sample (Supplementary Table S3 online). While the associations with longer reaction time on the K-CPT fell short of statistical significance, new associations emerged with ADHD symptoms, suggesting reduced symptoms with longer duration of any breastfeeding. There were still no significant associations with ADHD score in the clinical range or CAST score in the borderline range (not shown). 
Table 1. Descriptive characteristics of the study sample

\begin{tabular}{|c|c|c|c|c|}
\hline Variables & $N$ & Mean (SD) & Range & $\%$ \\
\hline \multicolumn{5}{|l|}{ Child characteristics } \\
\hline Age at testing (years) & 1,346 & $4.9(0.7)$ & $4.0-6.9$ & \\
\hline Sex & 1,346 & & & \\
\hline Female & 667 & & & 49.6 \\
\hline Birthweight (g) & 1,346 & $3297(433)$ & $1,825-4,800$ & \\
\hline Low birthweight $(<2,500 \mathrm{~g})$ & 38 & & & 2.8 \\
\hline Sub-cohort & 1,346 & & & \\
\hline Asturias & 299 & & & 22.2 \\
\hline Gipuzkoa & 188 & & & 14.0 \\
\hline Sabadell & 375 & & & 27.9 \\
\hline Valencia & 484 & & & 36.0 \\
\hline \multicolumn{5}{|l|}{ Maternal characteristics } \\
\hline Age at conception (years) & 1,346 & $31.0(4.0)$ & $17-43$ & \\
\hline$<25 y$ & 65 & & & 4.8 \\
\hline $25-29 y$ & 429 & & & 31.9 \\
\hline $30-34 y$ & 586 & & & 43.5 \\
\hline $35+y$ & 266 & & & 19.8 \\
\hline Parity at child's birth & 1,346 & $0.5(0.6)$ & $0-4$ & \\
\hline Parity $\geq 1$ & 560 & & & 41.6 \\
\hline Education level & 1,346 & & & \\
\hline Primary or less & 282 & & & 21.0 \\
\hline Secondary & 574 & & & 42.6 \\
\hline University & 490 & & & 36.4 \\
\hline Social class & 1,346 & & & \\
\hline $\begin{array}{l}\text { Managers/professionals/ } \\
\text { technicians }\end{array}$ & 545 & & & 40.5 \\
\hline $\begin{array}{l}\text { Skilled manual/other } \\
\text { nonmanual }\end{array}$ & 495 & & & 36.8 \\
\hline Semiskilled/unskilled & 309 & & & 22.7 \\
\hline Country of birth & 1,346 & & & \\
\hline Foreign & 82 & & & 6.1 \\
\hline Smoking at third trimester & 1,346 & & & \\
\hline Yes & 214 & & & 15.9 \\
\hline $\begin{array}{l}\text { Verbal IQ proxy (based on } \\
\text { WAIS-III Similarities) }\end{array}$ & 1,346 & $99.9(14.8)$ & $40-150$ & \\
\hline Psychopathology (SCL-90-R) & 1,346 & $49.9(10.0)$ & $35-69$ & \\
\hline $\begin{array}{l}\text { At risk (Global Severity Index } \\
\text { T-score }>65 \text { ) }\end{array}$ & 117 & & & 8.7 \\
\hline \multicolumn{5}{|l|}{ Paternal characteristics } \\
\hline Education level & 1,346 & & & \\
\hline Primary or less & 459 & & & 34.1 \\
\hline Secondary & 590 & & & 43.8 \\
\hline University & 297 & & & 22.1 \\
\hline
\end{tabular}

Table 1. Continued

\begin{tabular}{|c|c|c|c|c|}
\hline Variables & $N$ & Mean (SD) & Range & $\%$ \\
\hline Social class & 1,346 & & & \\
\hline $\begin{array}{l}\text { Managers/professionals/ } \\
\text { technicians }\end{array}$ & 411 & & & 30.5 \\
\hline $\begin{array}{l}\text { Skilled manual/other } \\
\text { nonmanual }\end{array}$ & 540 & & & 40.1 \\
\hline Semiskilled/unskilled & 395 & & & 29.3 \\
\hline \multicolumn{5}{|c|}{ Other environmental characteristics } \\
\hline Child care & 1,346 & & & \\
\hline Attending to kindergarten & 556 & & & 41.3 \\
\hline
\end{tabular}

$$
\text { at } 18 \mathrm{mo} \text { (\% yes) }
$$

Quality of family context (Etxadi Gangoidi Scale) ${ }^{a}$

\begin{tabular}{lccc}
$\begin{array}{l}\text { Stimulation of cognitive and } \\
\text { linguistic development }\end{array}$ & 337 & $60.4(19.0)$ & $0-95.5$ \\
$\begin{array}{l}\text { Stimulation of social and } \\
\text { emotional development }\end{array}$ & 337 & $77.2(11.0)$ & $39.2-100.0$ \\
$\begin{array}{l}\text { Organisation of social } \\
\text { context and physical } \\
\text { environment }\end{array}$ & 337 & $78.1(13.1)$ & $22.0-100.0$ \\
\hline
\end{tabular}

\section{Breastfeeding} (months ; \% yes)

Predominant breastfeeding $\quad 1,325 \quad 3.4(2.0) \quad 0.0-10.1 \quad 77.3$ (months ${ }^{\mathrm{b}}$; \% yes)

$\begin{array}{lllll}\text { Exclusive breastfeeding } & 808 & 2.8(2.0) & 0.0-6.1 & 76.6\end{array}$ (months ${ }^{\mathrm{b}, \mathrm{c}} ; \%$ yes)

\section{Child test scores}

McCarthy Scales of Children's Abilities

$$
\text { General Cognitive Index } \quad 1,331 \quad 100.8(14.7) \quad \text { 35-148 }
$$

Kiddie Continuous Performance Test

$$
\text { Omissions }
$$

$1,042 \quad 22.0(18.6) \quad 0-129$

False alarms

$1,042 \quad 21.7(11.0) \quad 0-49$

Hit reaction time (ms)

$1,041 \quad 721.1(135.8) \quad 385-1,302$

ADHD-DSM-IV

$\begin{array}{lccc}\text { Inattention } & 1,130 & 3.7(4.7) & 0-24 \\ \text { Hyperactivity-impulsivity } & 1,134 & 3.4(4.5) & 0-27 \\ \text { Total score } & 1,115 & 7.0(8.0) & 0-51 \\ \begin{array}{l}\text { Score in the clinical range } \\ \text { (diagnostic criteria) }\end{array} & 69 & & \end{array}$

\begin{tabular}{|c|c|c|c|}
\hline Total score & 1,187 & $6.1(3.2)$ & $0-20$ \\
\hline Score in the clinical range $(\geq 15)$ & 17 & & 1.4 \\
\hline $\begin{array}{l}\text { Score in the borderline } \\
\text { range }(\geq 12)\end{array}$ & 70 & & 5.9 \\
\hline
\end{tabular}

Childhood Autism Spectrum Test 


\section{Articles $\mid$ Boucheretal.}

Table 2. Linear regression analyses examining association between duration of breastfeeding (in months) and child outcomes

\begin{tabular}{|c|c|c|c|c|c|c|c|c|c|c|}
\hline \multirow[b]{3}{*}{ Outcome } & \multicolumn{5}{|c|}{ Any breastfeeding } & \multicolumn{5}{|c|}{ Predominant breastfeeding } \\
\hline & \multirow[b]{2}{*}{$N$} & \multicolumn{2}{|c|}{ Minimally-adjusted } & \multicolumn{2}{|c|}{ Fully-adjusted model } & \multirow[b]{2}{*}{$N$} & \multicolumn{2}{|c|}{ Minimally-adjusted } & \multicolumn{2}{|c|}{ Fully-adjusted model } \\
\hline & & $\operatorname{std} \beta$ & $\mathrm{B}(95 \% \mathrm{Cl})$ & $\operatorname{std} \beta$ & $\mathrm{B}(95 \% \mathrm{Cl})$ & & $\operatorname{Std} \beta$ & $\mathrm{B}(95 \% \mathrm{Cl})$ & $\operatorname{std} \beta$ & $\mathrm{B}(95 \% \mathrm{Cl})$ \\
\hline \multicolumn{11}{|l|}{ MSCA } \\
\hline $\mathrm{GCl}$ & 1331 & $0.09^{* *}$ & $0.26(0.12,0.40)$ & $0.07^{* *}$ & $0.19(0.06,0.33)$ & 1311 & $0.10^{* *}$ & $0.64(0.30,0.98)$ & $0.06^{*}$ & $0.37(0.05,0.70)$ \\
\hline \multicolumn{11}{|l|}{ K-СРT } \\
\hline Omissions (In) & 1042 & -0.04 & $-0.01(-0.02,0.00)$ & -0.03 & $-0.01(-0.02,0.01)$ & 1036 & -0.05 & $-0.02(-0.04,0.00)$ & -0.04 & $-0.02(-0.04,0.01)$ \\
\hline False alarms & 1042 & -0.01 & $-0.03(-0.16,0.10)$ & -0.01 & $-0.03(-0.16,0.10)$ & 1036 & 0.00 & $0.02(-0.28,0.31)$ & 0.00 & $0.01(-0.28,0.31)$ \\
\hline Mean RT & 1041 & 0.05 & $1.42(-0.14,2.98)$ & $0.06^{*}$ & $1.74(0.13,3.34)$ & 1035 & 0.04 & $2.58(-0.95,6.11)$ & 0.05 & $3.31(-0.31,6.92)$ \\
\hline \multicolumn{11}{|l|}{ ADHD-DSM-IV } \\
\hline Inattention (In) & 1126 & $-0.06^{*}$ & $-0.01(-0.02,0.00)$ & -0.04 & $-0.01(-0.02,0.00)$ & 1105 & -0.06 & $-0.02(-0.05,0.00)$ & -0.03 & $-0.01(-0.04,0.01)$ \\
\hline Hyperactivity (In) & 1130 & $-0.06^{*}$ & $-0.01(-0.02,-0.00)$ & -0.05 & $-0.01(-0.02,0.00)$ & 1111 & -0.03 & $-0.01(-0.04,0.01)$ & -0.02 & $-0.01(-0.03,0.02)$ \\
\hline Total (In) & 1111 & $-0.06^{*}$ & $-0.01(-0.02,-0.00)$ & -0.05 & $-0.01(-0.02,0.00)$ & 1092 & -0.06 & $-0.03(-0.05,0.00)$ & -0.04 & $-0.02(-0.04,0.01)$ \\
\hline \multicolumn{11}{|l|}{ CAST } \\
\hline \multirow[t]{3}{*}{ Total score } & 1183 & $-0.11^{* *}$ & $-0.07(-0.10,-0.03)$ & $-0.07^{* *}$ & $-0.04(-0.08,-0.01)$ & 1172 & $-0.10^{* *}$ & $-0.14(-0.22,-0.06)$ & $-0.06^{*}$ & $-0.08(-0.16,-0.00)$ \\
\hline & \multicolumn{5}{|c|}{ Exclusive breastfeeding } & & & & & \\
\hline & & \multicolumn{2}{|c|}{ Minimally-adjusted } & \multicolumn{2}{|c|}{ Fully-adjusted model } & & & & & \\
\hline Outcome & $N$ & $\operatorname{std} \beta$ & $\mathrm{B}(95 \% \mathrm{Cl})$ & $\operatorname{std} \beta$ & $\mathrm{B}(95 \% \mathrm{Cl})$ & & & & & \\
\hline \multicolumn{11}{|l|}{ MSCA } \\
\hline $\mathrm{GCl}$ & 796 & $0.10^{* *}$ & $0.69(0.23,1.15)$ & $0.07^{*}$ & $0.48(0.04,0.92)$ & & & & & \\
\hline \multicolumn{11}{|l|}{ K-CPT } \\
\hline Omissions (In) & 550 & -0.02 & $-0.01(-0.04,0.02)$ & -0.00 & $-0.00(-0.03,0.03)$ & & & & & \\
\hline False alarms & 550 & 0.02 & $0.09(-0.38,0.56)$ & 0.02 & $0.08(-0.40,0.57)$ & & & & & \\
\hline Mean RT & 549 & 0.07 & $4.92(-0.93,10.78)$ & $0.10^{*}$ & $6.65(0.57,12.73)$ & & & & & \\
\hline \multicolumn{11}{|l|}{ ADHD-DSM-IV } \\
\hline Inattention (In) & 695 & -0.03 & $-0.01(-0.05,0.02)$ & -0.01 & $-0.00(-0.04,0.03)$ & & & & & \\
\hline Hyperactivity (In) & 705 & -0.04 & $-0.02(-0.05,0.02)$ & -0.03 & $-0.01(-0.04,0.02)$ & & & & & \\
\hline Total (In) & 692 & -0.03 & $-0.02(-0.05,0.02)$ & -0.01 & $-0.01(-0.04,0.03)$ & & & & & \\
\hline \multicolumn{11}{|l|}{ CAST } \\
\hline Total score & 668 & -0.07 & $-0.10(-0.21,0.01)$ & -0.03 & $-0.04(-0.15,0.07)$ & & & & & \\
\hline
\end{tabular}

Standardized regression coefficient (std $\beta$ ) indicates the number of standard deviations of the outcome variable score change per standard deviation increase in breastfeeding duration, and its value is comparable to a correlation coefficient. Raw regression coefficient (B) with its $95 \%$ confidence intervals ( $95 \% \mathrm{Cl}$ ) indicates the amount of outcome variable point change per additional month of breastfeeding. Higher MSCA score indicates better performance. Higher CAST and ADHD-DSM-IV scores indicate more symptoms. Higher K-CPT scores indicate poorer performance. Minimally adjusted models controlled for child characteristics (age, sex, cohort, birthweight, and quality of assessment (MSCA only)), and full models additionally controlled for child care, maternal variables (parity at birth, age, education, social status, country of birth, smoking during pregnancy, verbal IQ proxy, psychopathology), and paternal variables (education and social class).

ADHD-DISM-IV, ADHD criteria of Diagnostic and Statistical Manual of Mental Disorders, Fourth Edition; CAST, Childhood Autism Spectrum Test; K-CPT, Kiddie Continuous Performance Test; In, natural logarithm; MSCA - GCl, McCarthy Scales of Children's Abilities - General Cognitive Index.

${ }^{*} P<0.01 * * P<0.05$

\section{Duration of Any Breastfeeding as a Categorical Variable}

Supplemental analyses using categories instead of a continuous variable of duration of any breastfeeding revealed that, in comparison to children breastfed 0 to $2 \mathrm{mo}$, those who were breastfed for more than 12 mo had a 3.65-point advantage (0.24 SD) on the MSCA GCI and had a 0.62-point reduction (0.19 SD) on the total CAST score (Supplementary Table S4 online). It should be noted that statistical control for socio-environmental covariates seemed to have a stronger effect for breastfeeding 6 to $12 \mathrm{mo}$ in comparison to breastfeeding $>12 \mathrm{mo}$, which may reflect a smaller association between these confounding factors and breastfeeding duration when breastfeeding extends beyond usual duration recommendations.

\section{Effect of Child Sex}

Analyses stratified by sex revealed somewhat stronger beneficial effects among girls, in whom associations with cognitive development and autistic traits were statistically significant. However, none of the interaction terms reached statistical significance (Table 3 ). 


\section{Control for Quality of Family Context}

Inclusion of quality of family context variables, within the subsample of participants from the Gipuzkoa sub-cohort in whom the EGS was used, had very little influence, if any, on the regression coefficients for the association between breastfeeding duration and child outcomes, suggesting that these variables were not a significant confounder in our sample (Supplementary Table S5 online).

\section{Independence of the Relationship Between Breastfeeding and Each Outcome}

Results from regression models including other outcomes as covariates suggest that the relationships of breastfeeding duration to cognitive development and to autistic traits are independent from each other and from ADHD symptoms (Supplementary Table S6 online).

\section{Impact of Missing Data}

Associations between the duration of any breastfeeding and child outcomes were re-examined in supplemental analyses using inverse probability weighting to deal with missing data (Supplementary Table S7 online). In these models, the relations of breastfeeding to cognitive development and to ADHD symptoms remained stable and were statistically significant. However, the effect on autistic traits was reduced to a nonsignificant trend $(P=0.098)$.

\section{DISCUSSION}

This study examined the relationship of breastfeeding with cognitive development, attention, ADHD symptoms, and autistic traits at $4 \mathrm{y}$ of age in a multi-center prospective birth-cohort study of Spanish children. Longer duration of breastfeeding was independently associated with a small improvement in global cognitive function and with slightly fewer autistic traits. Although part of this association was explained by environmental factors, results were robust as they remained statistically significant after control for a wide range of confounders. In fully adjusted statistical models, each additional month of any type of breastfeeding was associated with a 0.19-point increase in MSCA GCI and with a 0.04-point decrease in CAST total score. More mitigated effects were found on ADHD symptoms and attention function. Beneficial associations were more evident in girls, although there were no significant interactions between breastfeeding and child sex. Whether duration of any, predominant, or exclusive breastfeeding was used as the exposure variable had little influence on our results. Our results provide additional support for recommendations of prolonged breastfeeding duration to promote child development, and suggest that beneficial effects still occur with breastfeeding beyond the first 12 mo of life.

The positive association found between breastfeeding duration and intellectual performance is consistent with the majority of studies on the topic $(1,3-6)$. Although several of these studies have been criticized for their lack of documentation of socio-environmental confounders, our results suggest that this association is not entirely explained by such factors. Nevertheless, failure to adjust for these variables is most likely to result in an overestimation of the beneficial effects of breastfeeding, as supported by previous studies $(2,3)$. Indeed, in our study, statistical control for socio-environmental confounders

Table 3. Linear regression analyses examining associations between duration of any breastfeeding (in months) and child outcomes, according to child sex

\begin{tabular}{|c|c|c|c|c|c|c|c|}
\hline & \multicolumn{3}{|c|}{ Girls } & \multicolumn{3}{|c|}{ Boys } & \multirow{2}{*}{$\begin{array}{c}\begin{array}{c}\text { Breastfeeding } \times \\
\text { Gender interaction }\end{array} \\
P \text {-value }\end{array}$} \\
\hline & $N$ & $\operatorname{std} \beta$ & $\mathrm{B}(95 \% \mathrm{Cl})$ & $N$ & $\operatorname{std} \beta$ & $\mathrm{B}(95 \% \mathrm{Cl})$ & \\
\hline $\mathrm{GCl}$ & 663 & $0.10^{*}$ & $0.27(0.08,0.46)$ & 668 & 0.04 & $0.11(-0.09,0.31)$ & 0.42 \\
\hline \multicolumn{8}{|l|}{ K-CPT } \\
\hline Mean RT & 527 & 0.04 & $1.16(-1.17,3.49)$ & 514 & $0.10^{*}$ & $2.64(0.40,4.89)$ & 0.24 \\
\hline \multicolumn{8}{|l|}{ ADHD-DSM-IV } \\
\hline Inattention (In) & 552 & -0.01 & $-0.00(-0.02,0.01)$ & 574 & -0.06 & $-0.01(-0.03,0.01)$ & 0.25 \\
\hline Hyperactivity (In) & 556 & -0.02 & $-0.00(-0.02,0.01)$ & 574 & -0.07 & $-0.01(-0.03,0.00)$ & 0.27 \\
\hline
\end{tabular}

Standardized regression coefficient (std $\beta$ ) indicates the number of standard deviations of the outcome variable score change per standard deviation increase in breastfeeding duration, and its value is comparable to a correlation coefficient. Raw regression coefficient (B) with its $95 \%$ confidence intervals ( $95 \%$ Cl) indicates the amount of outcome variable point change per additional month of breastfeeding. Higher MSCA score indicates better performance. Higher CAST and ADHD-DSM-IV scores indicate more symptoms. Higher K-CPT sCores indicate poorer performance. All models controlled for child characteristics [age, cohort, birthweight, and quality of assessment (MSCA only)], child care, maternal variables (parity at birth, age, education, social status, country of birth, smoking during pregnancy, verbal IQ proxy, psychopathology), and paternal variables (education and social class).

ADHD-DISM-IV, ADHD criteria of Diagnostic and Statistical Manual of Mental Disorders, Fourth Edition; CAST, Childhood Autism Spectrum Test; K-CPT, Kiddie Continuous Performance Test; In, natural logarithm; MSCA - GCl, McCarthy Scales of Children's Abilities - General Cognitive Index.

${ }^{*} P<0.01 ; * P<0.05$ 


\section{Articles | Boucheretal.}

reduced the strength of this association by about $30-45 \%$, and the remaining effect, although statistically significant, was small and minimally clinically relevant. The relatively high proportion of mothers in our sample who reported breastfeeding their child for more than 12 mo may have contributed to these positive results. Beneficial effects of breastfeeding on cognitive development are especially salient among this group, possibly partly due to reduced confounding by measured socio-environmental factors when breastfeeding duration extends beyond recommendations.

The relationship of breastfeeding to ADHD behaviors and externalizing behavioral problems has been examined in a few prospective studies. In a previous study conducted in Menorca, our group reported decreased ADHD behaviors and better social competence as a function of breastfeeding duration, although this study offered no control for maternal IQ and psychopathology (10). In the present study, the relationship of breastfeeding to ADHD symptoms persisted after taking these confounders into account when we excluded nonbreastfed children from the analyses. The relationship was also significant in supplemental analyses using inverse probability weighting to deal for missing values. Other studies examining such behavioral problems have provided mixed findings. In a birth-cohort study conducted in Australia, breastfeeding for $\geq$ 6 mo was associated with decreased internalizing and externalizing behavior problems from infancy to adolescence in comparison to breastfeeding less than 6 mo (26). More recently, a large prospective study in United Kingdom found that, in comparison to not breastfeeding, breastfeeding for 2 to 4 mo was associated with decreased hyperactivity, and breastfeeding for $\geq 4$ mo was associated with decreased behavioural problems, as assessed with the Strengths and Difficulties Questionnaire at $5 \mathrm{y}$ in children born at term (9). However, other studies using this questionnaire did not replicate this finding, even though some had found a positive association with cognitive development $(4,11,12,27)$.

Our results do not support the presence of beneficial effects of prolonged breastfeeding duration on attention performance. Conversely, we found an association between longer duration of breastfeeding and slower reaction times on the K-CPT in some of our statistical models. Slower reaction times on the $\mathrm{K}-\mathrm{CPT}$ typically indicate slower processing speed, although with children as young as 4-6 y it may also be confounded by better impulse control. Such a poorer validity of the K-CPT with young children is supported by the weak correlations observed with parental and socio-environmental characteristics, which in some cases were in the opposite direction to what would be expected in older children and in adults.

To our knowledge, our study is the first prospective investigation to suggest that longer duration of breastfeeding protects against developing autistic traits, and our data indicates that this association is independent from cognitive performance. At least three case-control studies also support the hypothesis of an association between breastfeeding and ASD. A study using data from an internet survey involving 861 children with ASD and 123 controls found that children who were not breastfed were 2.5 times more likely to suffer from ASD in comparison to children breastfed more than 6 mo (14). A small parent survey of 60 children with and 85 children without ASD found that increased duration of breastfeeding was associated with a reduction in the number of ASD diagnoses (15). Another study involving 102 ASD children and 102 matched controls from Oman also reported decreased risk of ASD in relation to earlier initiation of breastfeeding, increased duration of exclusive breastfeeding, and continued breastfeeding (13). This contrasts with results from a recent study using data from the National Survey of Children's Health, a large nationally representative survey of US children including 37,901 children aged 2 to $5 \mathrm{y}$, which found no association between breastfeeding and ASD diagnosis (16). The very young age $(<3 \mathrm{y})$ of some children, combined with reliance on health care provider diagnosis, may have resulted in misclassification of ASD cases in this survey, preventing the finding of significant associations. The factors contributing to ASD are poorly understood. Although genetic causes are well established, concordance among monozygotic twins is less than $100 \%$ and the severity and range of symptoms among concordant twins often differ strikingly (28). This suggests that environmental factors are also involved, most probably through complex gene-environment interactions. Possible pathways for protective effects of breastfeeding against autistic traits include insulin-like growth factor deficiency, since it has been proposed as a possible factor in the development of autistic characteristics, which can be prevented by prolonged breastfeeding, and oxytocin release in the brain through breastfeeding stimulation $(15,29)$. However, caution should be paid to interpretation of our findings, as the association between duration of any breastfeeding and autistic traits fell short of statistical significance in supplemental analyses using inverse probability weighting to deal for missing values, and replication of these findings is warranted.

Among the most frequently cited mechanisms for beneficial effects of breastfeeding for neuropsychological development is maternal transfer of essential long-chain polyunsaturated fatty acids (LC-PUFAs), which are present in large quantities in breast milk (30). Higher intake of LC-PUFAs during early development has been associated with better cognitive function and decreased behavioral problems in children (31-33). LC-PUFAs thus constitute a plausible mechanisms of action through which breastfeeding promotes cognitive development and protects against ADHD symptoms. The LC-PUFA hypothesis has been tested within the subsample of our participants from the Sabadell cohort, for whom LC-PUFAs were assessed in colostrum. When children were tested during infancy, beneficial effects of breastfeeding were stronger with high LC-PUFA levels (34). However, when the same children were tested at 4 $y$, the relationship between breastfeeding and intelligence was independent of LC-PUFAs (5). Alternative hypotheses include physical or social interactions with the mother and influence of breastfeeding on gut microbiota (35). Further investigations are needed to understand how breastfeeding promotes brain development. 
Although our study supports the presence of protective effects of prolonged breastfeeding duration against autistic traits and, to a lesser extent, ADHD symptoms, these results should not be interpreted as an indication that breastfeeding prevents the development of autism or ADHD. Indeed, not only our associations with autistic traits and ADHD symptoms were relatively weak and had therefore limited clinical significance, but we also found no significant association between breastfeeding duration and the occurrence of scores within the clinical range. Although this may be related to the low incidence of such high scores in our sample, it is nonetheless congruent with the data from the US National Survey of Children's Health suggesting no association between breastfeeding and formal diagnosis of ASD (16). It is possible that a decrease in autistic traits does not prevent the development of ASD. Moreover, the design of our study does not allow to test for the causality of relationships. Since the child's behavior may influence the mother's motivation to pursue breastfeeding, we cannot rule out the possibility that infants who develop more autistic traits or ADHD symptoms during early childhood behaved in a way that incited their mothers to stop or reduce breastfeeding $(36,37)$.

Strengths of our study include a large sample size, prospective design, documentation of breastfeeding intensity, use of specific and validated measures of autistic traits and ADHD symptoms, and statistical control for a large number of potential confounders. The use of direct child assessments (MSCA and $\mathrm{K}-\mathrm{CPT}$ ) as well as parental (CAST) and teacher (ADHDDSM-IV) reports makes it unlikely that the beneficial effects reported here are the result of reporting bias, although attention assessment would have benefited from parental ratings as well. Despite the fact that particular attention was given to the issue of confounding, we cannot insure that we adjusted for all socio-environmental factors contributing to the association between breastfeeding and neuropsychological development. Another limitation is the relatively large child age range at assessment, which we addressed by controlling for child age in all of our statistical models.

\section{SUPPLEMENTARY MATERIAL}

Supplementary material is linked to the online version of the paper at http://www.nature.com/pr

\section{ACKNOWLEDGMENTS}

We would like to acknowledge the study participants for their generous collaboration, and the interviewers for their assistance in contacting families and administering the questionnaires.

\section{STATEMENT OF FINANCIAL SUPPORT}

This study was funded by grants from Spanish Ministry of Health-Instituto de Salud Carlos III (INMA Network G03/176, CB06/02/0041, FIS-PI041436, FIS- PI081151, FIS-PI042018, FIS-PI09/02311, FIS-PI06/0867, FIS-PS09/00090, FIS-PI03/1615, FIS-PI04/1509, FIS-PI04/1112, FIS-PI04/1931, FIS-PI05/1079, FIS-PI05/1052, FIS-PI06/1213, FIS-PI07/0314, and FIS-PI09/02647, CP14/00108), Generalitat de Catalunya-CIRIT 1999SGR 00241, Conselleria de Sanitat Generalitat Valenciana 1 (048/2010 and 060/2010), Universidad de Oviedo, Obra Social Cajastur, Department of Health of the Basque Government (2005111093 and 2009111069), the Provincial Government of Gipuzkoa (DFG06/004 and DFG08/001), and the Fundación Roger Torné. $\mathrm{O}$. Boucher was funded by post-doctoral grants from the Canadian Institutes of Health Research (MFE-115520). M.G. and J.J. were funded by a Miguel
Servet fellowship (MS13/00054, MS14/00108) awarded by the Spanish Institute of Health Carlos III (Ministry of Economy and Competitiveness).

Disclosure: Authors have no conflicts of interest or financial disclosures.

\section{REFERENCES}

1. Anderson JW, Johnstone BM, Remley DT. Breast-feeding and cognitive development: a meta-analysis. Am J Clin Nutr 1999;70:525-35.

2. Jacobson SW, Chiodo LM, Jacobson JL. Breastfeeding effects on intelligence quotient in 4- and 11-year-old children. Pediatrics 1999;103:e71.

3. Belfort MB, Rifas-Shiman SL, Kleinman KP, et al. Infant feeding and childhood cognition at ages 3 and 7 years. Effects of breastfeeding duration and exclusivity. JAMA Pediatri 2013;167:838-44.

4. Kramer MS, Aboud F, Mironova E, et al.; Promotion of Breastfeeding Intervention Trial (PROBIT) Study Group. Breastfeeding and child cognitive development: new evidence from a large randomized trial. Arch Gen Psychiatry 2008;65:578-84.

5. Julvez J, Guxens M, Carsin AE, et al. A cohort study on full breastfeeding and child neuropsychological development: the role of maternal social, psychological, and nutritional factors. Dev Med Child Neurol 2014;56:148-56.

6. Quigley MA, Hockley C, Carson C, Kelly Y, Renfrew MJ, Sacker A. Breastfeeding is associated with improved child cognitive development: a population-based cohort study. J Pediatr 2012;160:25-32.

7. Drane DL, Logemann JA. A critical evaluation of the evidence on the association between type of infant feeding and cognitive development. Paediatr Perinat Epidemiol 2000;14:349-56.

8. Jain A, Concato J, Leventhal JM. How good is the evidence linking breastfeeding and intelligence? Pediatrics 2002;109:1044-53.

9. Heikkilä K, Sacker A, Kelly Y, Renfrew MJ, Quigley MA. Breast feeding and child behaviour in the Millennium Cohort Study. Arch Dis Child 2011;96:635-42.

10. Julvez J, Ribas-Fitó N, Forns M, Garcia-Esteban R, Torrent M, Sunyer J. Attention behaviour and hyperactivity at age 4 and duration of breastfeeding. Acta Paediatr 2007;96:842-7.

11. Kramer MS, Fombonne E, Igumnov $S$, et al.; Promotion of Breastfeeding Intervention Trial (PROBIT) Study Group. Effects of prolonged and exclusive breastfeeding on child behavior and maternal adjustment: evidence from a large, randomized trial. Pediatrics 2008;121:e435-40.

12. Lind JN, Li R, Perrine CG, Schieve LA. Breastfeeding and later psychosocial development of children at 6 years of age. Pediatrics 2014;134 Suppl 1:S36-41.

13. Al-Farsi YM, Al-Sharbati MM, Waly MI, et al. Effect of suboptimal breastfeeding on occurrence of autism: a case-control study. Nutrition 2012;28:e27-32.

14. Schultz ST, Klonoff-Cohen HS, Wingard DL, et al. Breastfeeding, infant formula supplementation, and autistic disorder: the results of a parent survey. Int Breastfeed J 2006;1:16.

15. Shafai T, Mustafa M, Hild T, Mulari J, Curtis A. The association of early weaning and formula feeding with autism spectrum disorders. Breastfeed Med 2014;9:275-6.

16. Husk JS, Keim SA. Breastfeeding and autism spectrum disorder in the National Survey of Children's Health. Epidemiology 2015;26:451-7.

17. Guxens M, Ballester F, Espada M, et al.; INMA Project. Cohort profile: the INMA-INfancia y Medio Ambiente-(Environment and Childhood) Project. Int J Epidemiol 2012;41:930-40.

18. McCarthy, D. MSCA. Escalas McCarthy de Aptitudes y Psicomotricidad para Niños. Madrid: TEA Ediciones; 2009.

19. Conners CK, Staff M. Conners' Kiddie Continuous Performance Test (K-CPT): Computer Program for Windows Technical Guide and Software Manual. Toronto, ON: Multi-Health Systems, Inc; 2001.

20. American Psychiatric Association. Manual diagnóstico y estadístico de los trastornos mentales. Barcelona: Masson; 2002.

21. Fuentes, J., Pezzuto, C. Childhood Autism Spectrum Test (CAST) - Adaptación al español. Retrieved 2009. http://www.autismresearchcentre.com/ arc_tests.

22. Wechsler D, Kaufman A. WAIS-III. Escala de inteligencia de Wechsler para adultos (III). Madrid: Tea Ediciones; 2001.

23. Martínez-Azumendi O, Fernández-Gómez C, Beitia-Fernández M. [Factorial variance of the SCL-90-R in a Spanish out-patient psychiatric sample]. Actas Esp Psiquiatr 2001;29:95-102. 


\section{Articles | Boucher et al.}

24. Arranz E, Olabarrieta F, Manzano A, et al. Etxai-Gangoiti scale: a proposal to evaluate the family contexts of two-year-old children. Early Child Dev Care 2014; 184:933-48.

25. Seaman SR, White IR. Review of inverse probability weighting for dealing with missing data. Stat Methods Med Res 2013;22:278-95.

26. Oddy WH, Kendall GE, Li J, et al. The long-term effects of breastfeeding on child and adolescent mental health: a pregnancy cohort study followed for 14 years. J Pediatr 2010;156:568-74.

27. Belfort MB, Rifas-Shiman SL, Kleinman KP, et al. Infant breastfeeding duration and mid-childhood executive function, behavior, and socialemotional development. J Dev Behav Pediatr 2016;37:43-52.

28. Newschaffer CJ, Croen LA, Daniels J, et al. The epidemiology of autism spectrum disorders. Annu Rev Public Health 2007;28:235-58.

29. Steinman G, Mankuta D. Breastfeeding as a possible deterrent to autism-a clinical perspective. Med Hypotheses 2013;81:999-1001.

30. Brenna JT, Varamini B, Jensen RG, Diersen-Schade DA, Boettcher JA, Arterburn LM. Docosahexaenoic and arachidonic acid concentrations in human breast milk worldwide. Am J Clin Nutr 2007;85:1457-64

31. Helland IB, Smith L, Saarem K, Saugstad OD, Drevon CA. Maternal supplementation with very-long-chain n-3 fatty acids during pregnancy and lactation augments children's IQ at 4 years of age. Pediatrics 2003;111: e39-44.

32. Boucher O, Burden MJ, Muckle G, et al. Neurophysiologic and neurobehavioral evidence of beneficial effects of prenatal omega-3 fatty acid intake on memory function at school age. Am J Clin Nutr 2011;93: 1025-37.

33. Kohlboeck G, Glaser C, Tiesler C, et al.; LISAplus Study Group. Effect of fatty acid status in cord blood serum on children's behavioral difficulties at $10 \mathrm{y}$ of age: results from the LISAplus Study. Am J Clin Nutr 2011;94:1592-9.

34. Guxens M, Mendez MA, Moltó-Puigmartí C, et al. Breastfeeding, longchain polyunsaturated fatty acids in colostrum, and infant mental development. Pediatrics 2011;128:e880-9.

35. Parletta N. Breastfeeding and child neurodevelopment - a role for gut microbiota? Dev Med Child Neurol 2014;56:101-2.

36. Li R, Fein SB, Chen J, Grummer-Strawn LM. Why mothers stop breastfeeding: mothers' self-reported reasons for stopping during the first year. Pediatrics 2008;122 Suppl 2:S69-76.

37. Ahluwalia IB, Morrow B, Hsia J. Why do women stop breastfeeding? findings from the pregnancy risk assessment and monitoring system. Pediatrics 2005;116:1408-12. 